\title{
Efectos del prejuicio sexual en la salud mental de personas transgénero chilenas desde el Modelo de Estrés de las Minorías: Una aproximación cualitativa
}

\section{Sexual prejudice effects on Chilean transgender individuals' mental health from Minority Stress Model: A qualitative approach}

\author{
Jaime Barrientos Delgado \\ Facultad de Psicología, Universidad Alberto Hurtado, Chile \\ Ricardo Espinoza-Tapia \\ Escuela de Psicología, Universidad Católica del Norte, Chile \\ Patricio Meza Opazo \\ Escuela de Psicología, Universidad Católica del Norte, Chile \\ José Luis Saiz \\ Departamento de Psicología, Universidad de La Frontera, Chile \\ Manuel Cárdenas Castro \\ Escuela de Psicología, Universidad de Valparaíso, Chile \\ Mónica Guzmán-González \\ Escuela de Psicología, Universidad Católica del Norte, Chile \\ Fabiola Gómez Ojeda \\ Escuela de Psicología, Pontificia Universidad Católica de Chile, Chile \\ Joaquin Bahamondes Correa \\ School of Psychology, Faculty of Science, University of Auckland, Nueva Zelanda \\ Leonor Lovera Saavedra \\ Facultad de Psicología, Universidad Alberto Hurtado, Chile
}

Rec (01 de Julio de 2019) Acept (11 de Octubre de 2019)

\footnotetext{
Correspondencia: Jaime Barrientos, Facultad de Psicología, Universidad Alberto Hurtado- Email: jbarrientos@uahurtado.cl Agradecimientos: Agradecemos a organizaciones de la sociedad civil como OTD, Amanda Jofré y Arcoiris Trans Antofagasta. Asimismo, a la psicóloga Patricia Casanova, a la vez que a diversos informantes clave de la población transgénero, entrevistada en distintas regiones del país, que nos compartieron sus experiencias de vida, las cuales fueron fundamentales para el desarrollo de este trabajo de comunicación académica.

Este artículo fue financiado por la Comisión Nacional de Investigación Científica y Tecnológica (CONICYT) de Chile según el Proyecto FONDECYT № 1170046.
} 


\title{
Resumen
}

El Modelo de Estrés de las Minorías (Meyer, 2003) ha permitido explicar de qué forma el prejuicio sexual produce efectos negativos en la salud y bienestar de personas pertenecientes a las minorías sexuales, a través de la identificación de estresores de tipo distales y proximales. Este estudio buscó indagar los efectos del prejuicio sexual en la salud mental de personas transgénero en Chile desde un enfoque cualitativo. Se realizaron entrevistas semi-estructuradas a 17 personas transgénero femeninas y masculinas en cuatro ciudades del país. Los resultados nos permite identificar la presencia de factores distales asociados a discriminación manifiesta y factores proximales asociados a la vivencia del estigma y su relación con el autoconcepto. Finalmente, se describen efectos en la salud mental, entre los que destacan la presencia de sintomatología ansioso-depresiva, ideación e intentos suicidas, conductas autolesivas y consumo de alcohol y otras sustancias.

Palabras clave: Salud mental, prejuicio sexual, estrés de las minorías, transgénero.

\begin{abstract}
The Minority Stress Model (Meyer, 2003) has made it possible to explain how sexual prejudice produces negative effects on the health and wellbeing of people belonging to sexual minorities, through distal and proximal stressors. A qualitative study was conducted to investigate the effects of sexual prejudice on the mental health of transgender adults in Chile. Semi-structured interviews were conducted with 17 transgender people, both male and female, in four cities of the country. The results allow us to identify the presence of distal factors associated with overt discrimination, as well as, proximal factors associated with the experience of stigma and its effects on self-concept. Finally, effects on mental health are described, among which the presence of anxious-depressive symptomatology, suicidal ideation and attempts, self-injurious behavior and consumption of alcohol and other substances.
\end{abstract}

Keywords: Mental health, sexual prejudice, minority stress, transgender.

\section{Introducción}

Los derechos de las personas transgénero se encuentran en pleno debate en Chile actualmente, especialmente, luego de la aprobación de la ley anti-discriminación, conocida como "ley Zamudio" (Ley 20.609) en el año 2012 y, posteriormente, la ley de identidad de género (Ley 21.120). Esta última ley fue aprobada en el año 2018 y ha entrado en vigencia en diciembre del año 2019, posibilitando a personas transgénero adultas el reconocimiento de su identidad de género. Para el caso de las y los adolescentes, este derecho puede ser accesible, solo desde los catorce años y acreditando acompañamiento profesional junto con la aprobación de sus padres o cuidadores.

Sin embargo, pese a lo anterior, a nivel nacional aún persisten diferentes tipos de inequidades que afectan las relaciones de género y las sexualidades no-normativas (Barrientos, 2015). Dichas desigualdades son sostenidas por el patriarcado y el machismo, ambas ideologías asociadas a la dominación social y al privilegio que los hombres tienen sobre las mujeres en términos económicos, legales, judiciales, políticos, culturales y psicológicos (Barrientos, 2015).

Para Herek (2000), el patriarcado y el machismo favorecen la generación del prejuicio sexual, con las consecuencias negativas que este fenómeno tendría para las minorías sexuales y de género. Herek, Gillis \& Cogan (2009) definen el prejuicio sexual como las actitudes negativas hacia personas sustentadas en la orientación o identidad sexual de éstas. En esa línea, el autor sostiene que el prejuicio sexual casi siempre está dirigido hacia gays, lesbianas, bisexuales, personas transgéneros o intersexuales. El prejuicio sexual orientado hacia las personas transgénero se ha denominado transfobia. Además, en el caso de las personas transgénero, este prejuicio sexual se combina y profundiza con una actitud de rechazo hacia los cuerpos y el aspecto de las personas transgénero.

Para efectos del presente artículo usaremos la expresión prejuicio sexual, en vez de transfobia, ya que este primer término es el más consensuado en la investigación psicosocial sobre minorías sexuales y de género (Fraïsse \& Barrientos, 2016). Asimismo, utilizaremos el término transgénero para referirnos a aquellas personas 
cuya identidad de género o la expresión de éste difiere de lo esperado culturalmente, una vez hecha la asignación de sexo al nacer como hombre o mujer (Davidson, 2007; Valentine, 2007). Empero, si bien usamos dicho término, resulta relevante mencionar que las personas transgénero definen de muchas maneras su identidad de género (p.e, hombre, mujer, hombre transgénero, mujer transgénero, genderqueer, bigender, butch, queen) y la expresan en una enorme variedad de formas, las cuales dependen de cuestiones raciales/étnicas, socioeconómicas y lugar de residencia (Valentine, 2007). Además, emplearemos el término cisgénero para aludir a aquellas personas cuya identidad de género y el sexo asignado al nacer son concordantes con el comportamiento socialmente asignado (American Psychological Association [APA], 2015).

Respecto a la situación de personas transgénero, la evidencia científica señala que los niveles de prejuicio sexual hacia la población transgénero son más elevados que hacia gays, lesbianas o bisexuales (Barrientos, 2015; Brito, 2019; Comisión Interamericana de Derecho Humanos [CIDH], 2015). Por ejemplo, un estudio chileno indicaba que las personas transgénero femeninas, es decir, aquellas que hacen el tránsito de género de lo masculino a lo femenino, son particularmente susceptibles de sufrir niveles altos de prejuicio sexual, mayores que los reportados, en el mismo estudio, por hombres gays (Barrientos et al., 2016). Según el mismo estudio, el prejuicio hacia las personas transgénero es un serio problema en Chile y las personas transgénero femeninas perciben enormes desventajas frente a las autoridades, en el trabajo y a nivel institucional.

\section{Salud mental en personas transgénero}

El prejuicio sexual hacia personas transgénero puede entenderse como un tipo de experiencia de carácter negativo que posee efectos duraderos y acumulativos en la salud mental. Al respecto, la investigación sobre la salud mental en personas transgénero ha sufrido un enorme retraso debido a la clasificación de la transexualidad como trastorno mental por las ciencias psicológicas y médicas (Coll-Planas, 2010). Y, aunque el debate sobre el carácter patológico o no de las identidades transgénero per se aun no ha acabado, ello ha complicado la discusión e investigación sobre la salud mental de dicha población.

En base a las nuevas normas que se establecen en el Manual Diagnóstico y Estadístico de los Trastornos Mentales - V Edición (Diagnostic and Statistical Manual of Mental Disorders [DSM]), las personas transgénero sólo recibirán un diagnóstico de "disforia de género" cuando exista una incongruencia entre la propia experiencia de género y el género asignado. Pese a lo anterior, las asociaciones de personas transgénero han considerado que la despatologización de la transexualidad debe avanzar más allá de su remoción del DSM-V y otros manuales diagnósticos.

Otro aspecto del rezago en la atención a las necesidades particulares de la población transgénero es la falta de visibilidad que presenta en el plan chileno de salud mental y psiquiatría 2017-2025 (Ministerio de Salud de Chile, 2017), donde no existe referencia alguna a las problemáticas específicas de esta población, no obstante, dicho plan considera un enfoque de derechos y determinantes sociales de salud. Por el contrario, en otras regiones de latinoamérica, paulatinamente se han desarrollado guías de atención para profesionales de la salud mental orientadas al desarrollo de intervenciones que respeten los derechos de la comunidad lésbica, gay, bisexual y de personas transgénero (en adelante, LGBT), basadas en los principios de Yogyakarta, a la vez que la denuncia a las terapias reparativas de la homosexualidad y la patologización de la identidad transgénero (Oficina de las Nacionales Unidas contra la Droga y el Delito [UNODC], 2019).

Lo anterior, es relevante debido a que, según los datos entregados en América Latina por la Organización Panamericana de la Salud [OPS] (2016), los principales problemas de salud de la población transgénero son — o se derivan de-: a) altos niveles de exposición a violencia verbal, emocional y física incluyendo crímenes de odio; b) alta frecuencia de problemas relacionados con la salud mental (depresión y ansiedad), c) alta tasa de prevalencia de VIH y otras infecciones de transmisión sexual, d) alto consumo de alcohol y drogas, e) efectos negativos de hormonas autoadministradas, inyecciones de relleno de tejidos blancos y otras formas de modificación corporal, incluyendo complicaciones por malas intervenciones de reasignación de sexo y, f) problemas de salud reproductiva.

En Chile, datos recientes asociados a la salud mental de la población transgénero refieren el alto porcentaje de intentos de suicidios cometidos y altos niveles de discriminación percibida. Específicamente, en la primera encuesta nacional para estudiar diversos aspectos de las personas transgénero, conocida como "Encuesta T" (Organizando Trans Diversidades, 2017), un 56\% de los participantes declaró al menos un intento de suicidio. 
Dicho intento suicida fue reportado en un $48 \%$ entre los 11 a 15 años, mientras que un $27 \%$ lo reportó entre los 16 a 18 años. Por otra parte, entre los eventos de discriminación percibidos declarados como más frecuentes, están la discriminación en los centros de salud. En dicho contexto se reportó como eventos discriminatorios el "cuestionar la identidad", "sentirse ignorado" y las "burlas" (Organizando Trans Diversidades, 2017). Estos resultados, reafirman hallazgos reportados previamente por Berrero (2011) en una investigación realizada en Chile el año 2011, donde el $50 \%$ de la población transgénero señaló haber intentado quitarse la vida, mientras que casi la totalidad de los participantes del estudio manifestó haber sufrido depresión (87.5\%).

En esa misma línea, la OPS (2016) destaca que una característica común entre las personas transgénero durante su trayectoria vital es el aislamiento social, el cual puede causar ansiedad y depresión. Por otra parte, según el mismo informe, las preocupaciones sobre la aceptación social se transforman en un motivo constante de angustia, generando sufrimiento y aislamiento. Asimismo, en ese reporte se enfatiza la importancia que tiene la autoestima para una satisfactoria salud mental del colectivo transgénero.

Actualmente, a nivel internacional, se ha reportado vínculos entre identidades transgénero y altos niveles de desórdenes mentales (Dhejne, Van Vlerken, Heylens, \& Arcelus, 2016). Por tanto, se ha hipotetizado que la población transgénero está en mayor riesgo que la cisgénero y que otras minorías sexuales (gays, lesbianas y bisexuales) de experimentar mayores niveles de depresión, angustia, suicidio y otros desórdenes mentales, debido al mayor nivel de estrés social al que están sometidas (Bockting et al., 2016). Sobre este particular, Ilan Meyer (2003), desarrolló el Minority Stress Model (en adelante, MEM, por su definición en inglés), el cual ha sido traducido al español como "Modelo de Estrés de las Minorías".

\section{Modelo de Estrés de las Minorías (MEM)}

Ilan Meyer (2003) postula que para comprender los efectos del estrés en las minorías sexuales y de género debemos evaluar las múltiples fuentes de estrés social que afectan a dichos colectivos, incluyendo entre otras, eventos de discriminación, junto con las expectativas de rechazo, a la vez que la ocultación de la orientación sexual o la identidad y los mecanismos ligados a la homo/transfobia interiorizada. En dicho modelo se entiende por homo/transfobia interiorizada a todas aquellas manifestaciones de rechazo y discriminación hacia la propia identidad, junto con la evaluación negativa hacia aspectos de la diversidad sexual y de género. Asimismo, se le ha denominado interiorización del estigma (Calton, Bennett \& Gebhard, 2016).

Sintéticamente, para las personas transgénero, el MEM propone que añadir un estresor relacionado al prejuicio sexual, debido a su identidad, afectaría adversamente la salud y explicaría las diferencias de salud encontradas entre las personas transgénero y las cisgénero. Este tipo de estrés de las minorías es único dado que se suma a los estresores generales que son experimentados por todas las personas. Así, las personas discriminadas requieren un esfuerzo de adaptación superior al requerido por otras similares que no lo son. Además, es crónico, ya que es un tipo de estrés relativamente estable en el tiempo. Finalmente, está socialmente enraizado, es decir, se deriva de procesos sociales e institucionales, radicados en la estructura social, de difícil modificación, y por ello, más allá de las capacidades de las personas para cambiar las condiciones del entorno aversivo.

Según Meyer (2003), existen estresores distales, definidos como acontecimientos objetivos basados en procesos estructurales de la cultura, por ejemplo, eventos de discriminación y victimización hacia las personas LGBT, actitudes heterosexistas y rechazo a las minorías sexuales y de género. Por otro lado, los estresores proximales, son la extensión de los distales en la experiencia de las personas, vale decir, las percepciones individuales y apreciaciones relacionados con la propia identidad. Ejemplos de ello serían, la hipervigilancia, la transfobia internalizada, el ocultamiento de la identidad, la vivencia de estigma internalizada y las microagresiones producto de los procesos antes descritos (Tomicic et al., 2016).

Por tal motivo, en este artículo usaremos el MEM como soporte conceptual, ya que permite comprender el efecto del prejuicio sexual en la salud mental de las minorías sexuales y de género, siendo altamente utilizado a nivel global en diversos contextos en población gay, lésbica y transgénero (Bockting et al., 2016).

Lamentablemente, hasta la fecha existe poca evidencia a nivel nacional sobre la salud mental de la población transgénero. La mayor parte de la literatura existente corresponde a Estados Unidos y Europa, siendo escasos los estudios que documenten dicha asociación en América Latina (OPS, 2016). En Chile, entre los pocos estudios 
o propuestas existentes se destacan los trabajos realizados por el Movimiento Unificado de Minorías Sexuales [MUMS] (2009), Berredo (2011), Barrientos et al. (2016), Organizando Trans Diversidades (2017), Casanova \& Espinoza-Tapia (2018) y Tomicic et al. (2016).

Por lo que debido a la escasez de estudios que permitan conocer adecuadamente cómo afecta el prejuicio sexual en la salud mental de las personas transgénero chilenas, este estudio intenta responder a la siguiente interrogante: ¿Cómo es la salud mental asociada al prejuicio sexual desde la experiencia de personas transgénero adultas en Chile?

\section{Método}

\section{Diseño de investigación}

El estudio se enmarca en un paradigma comprensivo-interpretativo con un diseño cualitativo ya que es susceptible de caracterizar la subjetividad de las personas transgénero respecto de sus experiencias de prejuicio sexual percibidas y la relación con la identidad y la salud mental. El diseño y ejecución de este estudio contó con la colaboración de algunas agrupaciones transgénero del país y de informantes claves .

\section{Participantes}

La estrategia de selección de las personas participantes y construcción de la unidad de análisis fue mediante una estrategia de muestreo intencionado de variedad máxima, con el objetivo de obtener mayor riqueza de información por parte de los/as participantes (Patton, 2002). Finalmente, la unidad de análisis estuvo compuesta por un total de 17 personas, nueve que se definían como personas transgénero femeninas y ocho como transgénero masculinas, entre los 22 y 49 años de edad, pertenecientes a diferentes niveles socioeconómicos y educativos de las ciudades incluidas en el estudio: Antofagasta, Valparaíso, Santiago y Concepción. Para más detalle, ver tabla №1.

Dado que la población fue de difícil acceso, sobretodo las personas transgénero masculinas, la estrategia de muestreo incorporó los siguientes criterios de inclusión: (a) ser mayor de 18 años; (b) autodefinirse como transgénero femenina o masculino y, c) residir en las ciudades consideradas en el estudio.

A fin de conseguir heterogeneidad en las personas participantes, y considerando las dificultades de acceso a la población, el reclutamiento fue mediante el contacto con agrupaciones de personas transgénero e informantes clave, con quienes se orientó la búsqueda de personas transgénero con diversas trayectorias vitales ligadas a los siguientes criterios: nivel socioeconómico, generacional, educativo, activismo, ocupación, y asimismo, experiencias situadas en distintos contextos del país. Las personas que participaron recibieron una pequeña compensación económica por la participación en el estudio. 
Tabla 1. Caracterización de participantes

\begin{tabular}{|c|c|c|c|c|c|c|}
\hline Identidad & Edad & Ciudad & Activismo & Nivel Educacional & $\begin{array}{l}\text { Nivel } \\
\text { Socioeconómico }\end{array}$ & Ocupación \\
\hline $\mathrm{TF}$ & 49 & Antofagasta & No & Media incompleta & Medio bajo & Peluquería \\
\hline $\mathrm{TF}$ & 45 & Antofagasta & No & Media incompleta & Medio bajo & Peluquería \\
\hline $\mathrm{TM}$ & 22 & Antofagasta & Sí & Media completa & Medio & $\begin{array}{l}\text { Promotor empresa } \\
\text { retail }\end{array}$ \\
\hline TM & 23 & Antofagasta & Sí & Universitario & Medio & $\begin{array}{l}\text { Estudiante } \\
\text { universitario }\end{array}$ \\
\hline $\mathrm{TF}$ & 29 & Valparaíso & Sí & $\begin{array}{l}\text { Universitario } \\
\text { completa }\end{array}$ & Medio bajo & Emprendimiento \\
\hline $\mathrm{TF}$ & 45 & Valparaíso & No & Media completa & Medio bajo & $\begin{array}{l}\text { Construcción y } \\
\text { manicurista }\end{array}$ \\
\hline TM & 31 & Valparaíso & Sí & $\begin{array}{l}\text { Universitaria } \\
\text { incompleta }\end{array}$ & Medio bajo & Vendedor tienda \\
\hline TM & 29 & Valparaíso & No & $\begin{array}{l}\text { Universitaria } \\
\text { completa }\end{array}$ & Medio & Diseñador gráfico \\
\hline $\mathrm{TF}$ & 49 & Santiago & Sí & Básica & Bajo & Vendedora feria libre \\
\hline $\mathrm{TF}$ & 29 & Santiago & Sí & $\begin{array}{l}\text { Universitario } \\
\text { completo }\end{array}$ & Media bajo & $\begin{array}{l}\text { Cesante (Docente de } \\
\text { idioma francés }\end{array}$ \\
\hline $\mathrm{TF}$ & 49 & Santiago & No & Media completa & Media & Cantante y animadora \\
\hline $\mathrm{TM}$ & 27 & Santiago & No & Técnico superior & Media & Técnico en enfermería \\
\hline $\mathrm{TM}$ & 25 & Santiago & No & Universitario & Media & $\begin{array}{l}\text { Estudiante de } \\
\text { biotecnología }\end{array}$ \\
\hline $\mathrm{TF}$ & 30 & Concepción & Sí & Medio incompleto & Media & Comercio sexual \\
\hline $\mathrm{TF}$ & 26 & Concepción & Sí & $\begin{array}{l}\text { Universitario } \\
\text { completo }\end{array}$ & Alto & Fonoaudióloga \\
\hline $\mathrm{TM}$ & 27 & Concepción & Sí & $\begin{array}{l}\text { Universitario } \\
\text { incompleto }\end{array}$ & Media & Apoyo laboratorio \\
\hline TM & 28 & Concepción & Sí & Media completa & Medio bajo & Estación de servicio \\
\hline
\end{tabular}

Fuente: elaboración propia. TF: trangénero femenina; TM: transgénero masculino.

\section{Técnicas de producción de información}

La estrategia utilizada para la producción de información fue la entrevista semiestructurada. La información sociodemográfica se evaluó mediante preguntas abiertas, indagando en aspecto tales como: edad, sexo asignado al nacer, identidad de género y ocupación, entre otras. Lo anterior, derivó en una conversación con base en dos ejes temáticos: 1.- Identidad de género y proceso de afirmación de género; 2.- Prejuicio sexual y violencia manifiesta. Sobre este punto, las personas participantes respondieron preguntas abiertas, tales como: ¿Cómo defines tu identidad de género? ¿Cómo describirías tu proceso de afirmación de género? ¿Cómo ha sido tu estado de ánimo durante este proceso?

Las entrevistas fueron realizadas por integrantes del equipo de investigación, todos parte de la población LGBT. Estas se llevaron a cabo en diversos lugares, intencionando un espacio seguro que permitiera la libre expresión de las personas participantes. La duración fluctuó entre cuarenta y cinco minutos a una hora y media, aproximadamente. Las entrevistas fueron grabadas y posteriormente transcritas de forma literal para su análisis. 


\section{Estrategia de Análisis de información}

La información fue analizada bajo supuestos contemporáneos de la Teoría Fundamentada (Glaser \& Strauss, 1967), vale decir, la integración analítica de elementos de significado de los/las participantes, el marco teórico del estudio y los criterios de búsqueda relevantes para los investigadores (Charmaz, 2006), lo cual permitió organizar de forma estructurada la información emergente.

Con base en los supuestos del método, luego de lecturas de los relatos por parte del equipo de investigación, se realizó un proceso de codificación abierta y selectiva, en el que se identificaron categorías analíticas presentes en todas las entrevistas. Posteriormente, estas fueron contrastadas y organizadas según su pertenencia categorial y verificadas por parte de los investigadores según su dimensión de significado (Pérez Serrano, 1998). Los investigadores utilizaron los principios de "reflexividad" y "verificación intersubjetiva", documentando cuidadosamente cada paso del proceso de análisis, a la vez que acuciosos de la triangulación a nivel interpretativo.

\section{Criterios éticos}

Los criterios éticos de voluntariedad, confidencialidad y anonimato, fueron esenciales para este estudio. El comité de ética de la Universidad de Santiago aprobó el diseño del estudio previo al proceso de producción de datos. Las cartas de consentimiento por escrito fueron firmadas por cada persona entrevistada, según los requisitos de la Comisión Nacional de Investigación Científica y Tecnológica (CONICYT). Con el objetivo de preservar la confidencialidad de las personas participantes, la siguiente tabla expone el tipo de codificación utilizada para la caracterización de los relatos (ver tabla 2). A continuación, se presenta un ejemplo de codificación, Trans femenina, 49 años, Antofagasta: (TF (49) AFTA).

\section{Tabla 2. Codificación}

TF: Trans femenina - TM: Trans masculino. $\left(\mathrm{N}^{\circ}\right)$ : Edad.

AFTA: Antofagasta - VAL: Valparaíso - STGO: Santiago - CON: Concepción

Fuente: elaboración propia

\section{Resultados}

Para efectos de este artículo, los principales hallazgos fueron organizados de acuerdo con los supuestos del Modelo de Estrés de las Minorías en las siguientes dimensiones: 1.- Estresores distales; 2.- Estresores proximales; 3.- Efectos del prejuicio sexual en la salud mental.

\section{Estresores distales}

Los estresores distales se caracterizan por procesos y dinámicas presentes en la cultura que determinan la perpetración de violencia y discriminación hacia las personas transgénero. Esta violencia, identificada según el MEM como maltrato manifiesto, da cuenta de altos niveles de prejuicio sexual hacia las personas transgénero en la población general. Por ende, este tipo de violencia es ejercida por personas que configuran el entorno de las personas transgénero u otros contextos en los que se desenvuelven. Dichos actos de violencia atentan contra su integridad, provocando así que ellas vivan en una constante evaluación, alerta y cautela respecto de sus interacciones cotidianas.

En este contexto, el prejuicio sexual se asocia a las representaciones que la población tiene de aquellas personas cuya identidad de género difiere del binarismo heteropatriarcal (personas transgénero o de género fluido), como así también, a conductas negativas y agresivas perpetradas en contra de estas. 
Esta categoría agrupa diversas violencias reportadas por las personas transgénero, quienes expresan haber sido violentadas en muchas ocasiones a lo largo de sus vidas. Respecto a los contextos en los que ocurre el maltrato, estos son múltiples. Por ejemplo, en la familia de origen, con independencia del nivel socioeconómico y cultural, se evidencian formas activas de prejuicio sexual. Lo cual, podemos constatar en el siguiente relato. "Mi vieja me empieza a hacer una persecución satánica de que tengo que ser femenina (...) Me empieza a sacar las cejas, me obliga a vestirme más niñita. Ya no podía elegir mi ropa, me la elegían ellos. Me obligaron a dejarme crecer el pelo, me lo tiñó rojo" (TM (28) CON).

Estas expresiones de prejuicio en la familia y colegio, se inician con el cuestionamiento y rechazo de la identidad de género, confundiendola regularmente con un tipo de expresión de género ligada a la homosexualidad. Estas vivencias de hostilidad y agresiones generan sentimientos de inadecuación en la persona que las recibe. "Cuando era chico, vivía con mi abuela y era súper marimacho (...) andaba siempre escalando muros y me miraban feo porque me juntaba con niños o andaba con palos, jugando con pistolas y siempre era el niño de los juegos. Nunca la niñita o señorita... siempre me miraban feo, me decían: ";ay! ahí viene la (nombre femenino), la marimacha, la tres cocos"”, (TM (22) AFTA).

En otras palabras, existe a nivel social un cuestionamiento y rechazo hacia la expresión de género disidente de la heterosexualidad, lo cual desencadena una escalada de violencia, manifestada a través de sanciones que van desde la denostación, la ridiculización, la patologización y los castigos físicos, hasta la expulsión del hogar. "Cuando mi papá me echó de la casa, me fui donde una tía y esa tía estuvo todos los días sermoneándome sobre lo horrible que era mi condición y sobre lo muy alejada que estaba de Dios. Por lo menos me prestó una pieza para dormir" (TF (26) CON).

Las dinámicas descritas para el ámbito familiar se desplazan al contexto escolar, articulándose un escenario de hostigamiento constante. De esta manera, las primeras interpelaciones respecto de la no adecuación al modelo normativo binario de género provienen principalmente desde sus pares, donde la manifestación más común es el bullying. "Entré al colegio y ahí la sufrí caleta ... el bullying, todas las cosas juntas. Y yo, por dentro, siempre mal, sufría caleta, no sé poh, sufrí mucho, mucho. Sufría también en las micros, sufría mucho" (TM (29) VAL). En ese sentido, el rol de profesores y otras autoridades resulta significativo ya que ellos pueden ser parte activa de dichas prácticas violentas. "Mi profesora jefe me odiaba y andaba inventando que quería convertir en lesbiana a todo mundo" (TM (28) CON).

En el contexto laboral, las personas transgénero entrevistadas señalan que existen diversas vulneraciones. La principal forma consiste en la discriminación, lo cual les genera una importante precariedad económica. Así, algunas buscan alternativas para la subsistencia, principalmente en empleos informales y precarios. "Yo no he logrado tener a mis 30 años un trabajo. Porque, lo he tratado de conseguir, al momento de (...) paso todos los filtros típicos que te ponen en una entrevista laboral y me dicen: "si, estás muy apta para el trabajo, pero ahora me gustaría que me pases tu identificación para firmar contrato". En ese momento es como... "no me habias puesto al tanto de esto y creo que vamos a ver si en realidad hay un puesto, pero te llamamos"," (TF (30) CON).

En relación con lo anterior, las personas transgénero femeninas se dedican a labores tales como servicio doméstico, de limpieza y comercio sexual. "Me tengo que seguir dedicando a eso (trabajo sexual) porque no hay pega para trans" (TF (29) VAL). Para el caso de los trabajos formales, ellas se desempeñan en el área de servicios y peluquería. En cambio, las personas transgénero masculinas poseen mayores posibilidades de conseguir trabajo en áreas de servicio, aseo industrial y empresas de retail.

Respecto al contexto sanitario, la atención recibida en los centro asistenciales primarios o secundarios reproduce la hostilidad y cuesta enfrentarse a él, a pesar de que existen en el país instructivos específicos que regulan el respeto del nombre social y la identidad en el sistema de salud. "En salud, los médicos son una mierda. Y también es discriminación para mí, que yo haya tenido tanto miedo, durante tantos años de ir al médico" (TM (28) CON).

En específico, el abordaje por parte de los distintos integrantes de los equipos de salud (desde personal administrativo hasta los profesionales de salud), se caracteriza por el desconocimiento y un trato inadecuado y violento. "Fui a urgencias ginecológicas y no me querían atender ... porque yo fui y dije: "Hola, tengo un sangrado vaginal" (...) Me dijo: "présteme su carnet" y le paso mi carnet y me dice: "no, pero es que cómo va a tener un sangrado vaginal si usted es hombre". Le dije "yo no soy hombre, yo estoy operada, yo tengo vagina, yo tengo mi sangrado y por favor quiero que me atiendan. Entiendo tu confusión porque no es usual 
esto, pero esto es”" y me dijo: "pero es que estamos en ginecología ¿Cómo voy a anotar el nombre de un hombre en la ficha?" (TF (26) CON).

En la relación entre las personas transgénero y las instituciones públicas y privadas se identifica "violencia institucional", ya que — de acuerdo a sus relatos - refieren excesiva violencia. Además, las personas entrevistadas vivenciaban que no tienen muchas posibilidades de exigir cambios en la relación de ellas con dichas instituciones. Por tanto, asumen con cierta resignación tales vulneraciones. "Creo que una de la violencia más significativas fue esa violencia de la policía... que no recibe la denuncia, que me culpa a mí... que no hay cómo acogerte, no hay nada" (TM (27) CON).

Esta dinámica operaría con formas de agresión explícitas e implícitas, como es el caso de negar la atención o servicio, exponer públicamente el nombre jurídico y sexo asignado al nacer. Pero también, invisibilizar la versión de los hechos de violencia recibidos, minimizándolos o desconfirmándolos, lo cual configura el fenómeno de victimización secundaria. "Teníamos que ir a la Posta Central, donde el director era homofóbico (...) nos decian: "Oigan, aquí estamos pa' atenderlas no más, si tienen problemas con los pacos, vayan a hueviar pa' afuera”. De esa forma nos atendian (...) a algunas (trans) las habian matado a palos, y ellos le ponían en el documento cosas simples" (TF (49) STGO).

Respecto a los tipos de violencia percibida y recibida, la violencia física es definida por las personas participantes como aquella vulneración de la integridad física mediante golpes y/o empujones, o bien, la limitación de sus movimientos para que no puedan defenderse de la agresión. En muchas ocasiones, la violencia es ejercida con excesiva crueldad. "Yo vi a transgéneras que las dejaban en puros calzones, que les cortaban la cara, ¿cachai? Eh, después me fui dando cuenta de la crueldad de carabineros, de la policía de investigaciones. Nosotros me acuerdo de que teníamos que comprarle pollo asado, botellas de pisco para que no nos molestaran" (TF (49) STGO).

Para el caso de la violencia psicológica, esta es significada como cualquier acto que daña la estabilidad psicológica y emocional. Algunas de las formas en las que esta violencia se manifiesta es mediante insultos, humillaciones, desprecios, negligencia, ridiculización o amenazas de muerte. "La discriminación y los límites que me ponen los he sentido al momento de ir a hacer un trámite, al querer sacar una tarjeta de crédito. Ahi he sentido la discriminación, la he sentido mediante burlas, mediante caras extrañas al momento de identificarme con mi cédula" (TF (28) CON). De igual forma que la violencia física, la de tipo psicológica es referida con un alto impacto para el autoconcepto. "El dueño del lugar me mandó a decir que no volviera. Hubo gente que me eliminó de sus redes sociales, de todo contacto y nunca más me habló” (TF (26) CON).

También, existen otras manifestaciones de violencia como es la violencia simbólica, la cual se relaciona con las creencias referidas hacia las personas transgénero. Estas se manifiestan a través de formas verbales y no verbales. De modo que la violencia simbólica opera mediante creencias relativas a que ser transgénero es una alteración, una perversión o una enfermedad mental. Asímismo, este tipo de violencia opera mediante la normalización e incluso la justificación de cualquier tipo de violencia en contra de las personas transgénero: desde el no reconocimiento de la identidad, pasando por la ridiculización y patologización, hasta llegar a los golpes y el asesinato (crimen de odio). "Iba con ropa que yo decía que era ropa de hombre, pero me mandaban al baño de mujeres. De hecho, una vez que entré al baño de hombres, pero así con una ropa muy neutra, un señor se fue a quejar con la encargada porque dijo que había una mujer en el baño" (TM (29) VAL).

Igualmente, dicha violencia vincula a las personas transgénero con la prostitución, la anormalidad y un estilo de vida escandaloso y excesivo. En otras palabras, esta violencia las estigmatiza, provocando que las personas transgénero no puedan desarrollarse, ni desenvolverse en la sociedad tal como el resto de las personas. "Una persona trans quiere vivir y por eso hace lo que hace, porque quiere ser parte de la sociedad. Quiere integrarse, quiere vivir una vida normal... Pero lamentablemente termina viviendo una vida anormal, ya que la sociedad no le deja vivir una vida normal siendo trans" (TF (49) STGO).

En ese sentido, persisten representaciones y creencias en torno al motivo por el cual una persona transgénero realiza su proceso de afirmación de género. "Nos encuentran depravados por algún motivo. Porque parecen tener la convicción de que nuestra transición se debe a algo sexual y no tiene nada que ver con eso. No es sexual, es de identidad" (TF (26) CON). Además, las personas entrevistadas aluden a una popular creencia respecto a que se llega a ser transgénero producto de un trauma ligado a abuso sexual o maltrato en la infancia. "Porque otra generalización que se hace frente a las personas que son transgénero, trans, travesti, es que la mayoría 
de estas personas tenemos algún tipo de tranca psicológica, psiquiátrica ya sea por violación, abuso, ya sea no sé, padres quizá alcohólicos o drogadictos" (TM (27) CON).

\section{Estresores proximales}

Los estresores proximales corresponden a un conjunto de dinámicas y disposiciones ligadas a la identidad y a las percepciones de las personas transgénero basadas en los factores distales de estrés. Lo anterior, va a moderar la vivencia psicológica y expresión emocional, como así también, el desarrollo del autoconcepto y la afirmación de la propia identidad.

De acuerdo con los antecedentes expuestos en la categoría previa, el entorno es significado por parte de las personas transgénero como hostil y aversivo, de cara al reconocimiento y aceptación de aquellas identidades y orientaciones no heteronormadas. Por la misma razón, la apertura de ellas hacia el contexto social es un proceso lento, en el cual, aspectos tales como la orientación y/o identidad sexual comienzan a manifestarse progresivamente. "Y ahi, hay como varios ciclos entre medio, entre que me asumi gay a los 18 , decido ser completamente afeminado a los 23 y después a los 28 asumiendome trans" (TF (29) STGO).

Existiría una definición cis-heteronormativa de la identidad trans. Esta afirmación supone la existencia de una matriz de creencias y prácticas ligadas a la dicotomía sexo/género que se reproduce en nuestra cultura en función del mantenimiento de actitudes, creencias y comportamientos reiterativos en el diario vivir de la población. Así es como esta configuración de características vinculadas al binarismo de género persiste, incluso en niveles menos conscientes, normalizando comportamientos y legitimando solo dos formas únicas de ser: hombre y mujer; la heterosexualidad como norma y la concordancia entre la identidad de género y la experiencia corporal-genital.

Este proceso involucra a personas de la diversidad sexual $-\mathrm{y}$ en particular - a las personas transgénero quienes no pueden escapar de identidades de tipo dicotómicas y sus respectivas formas de expresión y expectativas ligadas a esos roles, tratando de identificarse finalmente con dos seres imaginarios a los que se aspira ser: "el hombre masculino v/s la mujer femenina". "Yo decía, para mi (...) me sentía mujer, entonces eso... me sentía más mujer. Porque estaba con pura gente hetero" (TF (49) AFTA).

Acorde a lo expresado por las personas participantes, las identidades transgénero no se alejan completamente de este modelo binario. Por lo mismo, la idea de "sentirse mujer" o "sentirse hombre" es atravesada por estas conceptualizaciones preconcebidas y reproducidas bajo estas mismas estructuras. Lo anterior, evidencia el lugar de dominación que ocupa la heteronormatividad y las experiencas CIS, sobre las experiencias trans, de manera que se crea una relación de subordinación desde lo cisgénero hacia lo transgénero. "Siempre me he sentido un hombre con pene, ¿cachai? Yo ahora sé que hay hombres que se sienten hombres con vagina. Ponte tú he leido harto, no me siento en absoluto representado por eso, para nada. Yo me siento un hombre con pene, con testículo" (TM (29) VAL). En relación con este punto, las vivencias transgénero no escapan del modelo sexo/ género binario, configurándose un relato sobre la identidad que responde a roles de género estereotipados y reducidos a la relación entre la identidad de género con la genitalidad.

Las mujeres transgénero entrevistadas tienden a definir su identidad de género desde la estructura heterocisnormativa. En particular, aquellas de mayor rango de edad son críticas de las personas transgénero que transgreden la relación entre identidad de género y orientación sexual. "Uno tiene que definirse al final, poh. Aunque uno pueda tener las cosas que uno tenga, pero uno tiene que definirse, poh. Pa' donde va tu micro" (TF (49) AFTA). Quizás por ello se sanciona discursivamente a aquellas transgénero femeninas que no poseen una orientación sexual heterosexual luego del proceso de afirmación de género, existiendo la expectativa de que se vinculen con hombres heterosexuales en sus actuales relaciones de pareja.

Para el caso de los hombres transgénero entrevistados, ellos perciben la influencia del modelo sexo/género predominante, identificando en sus prácticas mandatos atribuidos socialmente a la masculinidad hegemónica. Esta expectativa del rol ubica a los hombres en espacios productores de bienes materiales y responsables económicamente de sus familias, mientras que a las mujeres se les asigna al espacio privado del hogar. "Y ahí empezó la otra tortura, porque esta familia que nos acoge trata de imponer este nuevo estereotipo de género, entonces me prohiben tener amigos, salir los fines de semana. Tenía que ser sólo este hombre proveedor para juntar la plata y así sacar adelante a mi pareja, o sea, todos los estereotipos clásicos de toda la vida" (TM (28) CON). 
Por ende, en la búsqueda del reconocimiento de su propia identidad de género, las personas transgénero se ven constantemente reguladas en su subjetividad por expectativas ligadas a estereotipos de género de tipo binario. "Ahora mi aspecto me permite pasar piola y la gente que yo tengo de antes la he conservado porque no tiene problemas conmigo. Pero, aun asi, hay algunas personas, que yo noto en algunos comentarios, me ven como una mujer con un pero... una mujer entre paréntesis” (TF (26) CON).

En otro sentido, la visibilidad y el reconocimiento de la identidad transgénero se somete a un proceso heteronormado que se manifiesta en la subjetividad de las participantes. Por ello, es posible identificar manifestaciones tales como la negación y el ocultamiento de la identidad, lo cual se inicia en el entorno familiar y escolar, producto del desconocimiento y estigma asociado a las minorías sexuales y de género. "Yo no sé si realmente he terminado de lograr superar todas las dificultades que implican ser transgénero, pero siento que incluso en mi casa hasta el día de hoy ha sido una pelea constante” (TF (29) STGO). Además, resulta altamente frecuente la creencia ligada a que la persona transgénero sería homosexual o lesbiana, lo cual incide en la construcción de la propia identidad, generando confusión y malestar. "Me pusieron en colegio católico. Por lo tanto, demostrarme de una manera masculina, como yo era, frente a mis compañeros, frente a mis profesores, fue problema varias veces. Mi mamá tuvo que ir varias veces porque mis conductas no correspondian, obviamente, al género que se me asignó al nacer (...) fue triste porque yo sabía que no podía hacer nada porque no tenía voz, porque era pequeño, porque nadie me iba a escuchar, porque mi familia no tenía las herramientas, no tenían los conocimientos tampoco" (TM (27) CON).

Con base en lo anterior, el ocultamiento de la identidad es una estrategia que permite regular y anticipar la ocurrencia de algún acto aversivo por parte de su entorno. "Algunas veces sí, claro, evito hablar del tema, pero por eso digo que me presento siempre como (nombre femenino), y luego, si veo alguna reacción, pregunto también: “¿tú tienes complejidad con estas cosas?”, y luego de eso, aclaro que soy trans y me hago llamar (nombre masculino)" (TM (23) AFTA).

Otra línea de hallazgos consiste en la identificación de mecanismos de estigma sexual internalizado, mediante procesos tales como la transfobia internalizada y la normalización de la violencia. Para el caso de la transfobia internalizada resulta significativo identificar que las manifestaciones de violencia descritas por las personas participantes no vendrían solamente desde personas heterosexuales, sino que también serían expresadas por personas de la población LGBT. Igualmente, la violencia provendría de la internalización del prejuicio, y por ende, las personas transgénero manifestarían rechazo hacia otras personas transgénero de su misma comunidad. "Estas se ponen en la esquina, putean, no sé... ganan plata, no tienen la voluntad de hacer algo" (TF (49) STGO).

En el relato previo se muestra cómo las personas transgénero se observan y rechazan aspectos de sí mismas y de su propia comunidad, describiendolas como conflictivas, delincuentes, borrachas o con una expresión inadecuada de su identidad. "Yo también me voy a incluir, somos medias borrachitas y faltamos un poco el respeto" (TF (49) AFTA).

Un hallazgo significativo para la presente investigación consistió en la identificación de normalización de la violencia recibida y percibida por parte de las personas transgénero, generando una suerte de naturalización y minimización de tales violencias. Inclusive, las personas pueden llegar a preferir experimentar cierto tipo de violencia por sobre otra. "Obviamente me siento mucho más... no cómoda, pero más identificada con recibir esta violencia (verbal) a recibir la otra” (TF (26) CON).

Las personas entrevistadas justifican dicha opción previa debido a que creen que están en tensión con el modelo dominante de sexo/género, por lo que deben asumir dicho sacrificio a lo largo de sus vidas. "No he vivido grandes problemas de violencia ni discriminación totalmente arbitraria, a los más un par de empujones, un par de groserías o ataque verbal, pero en mi caso, menos mal, que no ha sido más que eso"” (TM (27) CON).

La minimización operaría cuando las personas transgénero no logran visualizar la existencia de conductas y/o comportamientos violentos hacia su persona, o bien, justifican que determinados actos de violencia ocurran, disminuyendo la importancia y/o naturalizándolos. "Si te pegaban, te lo merecí por estar hueviando poh... entonces era súper complicado... la que tenía la patudez o la osadía de salir pintá a la calle (silencio)... no sabíai si volvíai, tan simple como eso" (TF (48) STGO). Esta justificación, hecha por las propias personas transgénero, se basaría en el argumento de que toda vez que una persona desafía la heteronorma y el binarismo de género, debería asumir que podría ocurrir algún tipo de sanción desde dicha hegemonía. 


\section{Efectos del prejuicio sexual en la salud mental}

El prejuicio sexual contribuye, tal como lo anticipa el MEM, a la aparición de efectos negativos en la salud mental en las personas transgénero y puede llegar a provocar, ansiedad, depresión, ideación suicida, intentos de suicidio y suicidio, conductas autolesivas y consumo de sustancias (Clements-Nolle, Marx, Guzmán \& Katz, 2001; Mathy, 2002; OPS, 2016).

De modo que los estresores distales y proximales revisados en los apartados previos se configuran en factores de riesgo que podrían estar a la base de problemas de salud mental. Estos factores revelan el impacto psicológico del prejuicio sexual. Dicho análisis puede sintetizarse e ilustrarse a través del siguiente relato. "Todo ha sido muy estresante... mi nivel de salud mental estuvo permanentemente bajo, durante todos esos años. Estuve peligrosamente cerca del suicidio. Lo reconozco ahora... en ese momento yo no lo quería reconocer, pero yo pensaba mucho en el suicidio. Bajé mi rendimiento en la universidad claramente - pero bueno-yo siempre fui muy estudiosa, entonces, al final igual saqué la carrera al día, más que nada hostigada por el látigo de saber que mientras no terminara mi carrera, no me iba a poder independizar de mi familia y no iba a poder transicionar" (TF (26) CON).

A diferencia de la población cisgénero, en este estudio observamos como la percepción de constante amenaza a ser sancionado, excluído, interpelado y evaluado socialmente por la identidad de género, se vuelve una fuente de estrés cotidiana en la población transgénero. Con base en lo anterior, es posible de identificar un estado de alerta constante, unido al recuento y posterior clasificación de las experiencias potencialmente peligrosas para las personas transgénero.

Es decir, debido a la cotidianeidad con la que las personas transgénero son víctimas de burlas, miradas evaluativas, comentarios negativos, insultos y empujones, van creando sus propios parámetros de aceptación personal respecto a las violencias que pueden o no soportar. Tal como lo podemos apreciar en el siguiente relato. "Yo creo que mientras no te digan algo en la calle, mientras no te miren de una manera quizás mal, porque el comentario... como digo... siempre va a estar, pero mientras no pase de eso, siento que no hay tanto problema" (TF (20) VALPO).

Lo anterior, resulta un elemento importante del MEM, ya que las expectativas de rechazo que presenta esta población conllevan la aparición y cronicidad de diversa sintomatología y trastornos de salud mental que pasamos a comentar.

La sintomatología ansiosa puede llegar a fragilizar significativamente la salud mental de las personas transgénero. Lo anterior, es descrito en las entrevistas como la ansiedad ante diversos tipos de situaciones sociales a las que se ven enfrentadas y sus consecuencias. "Y efectivamente tengo flashbacks, siento mucha ansiedad ante ciertas situaciones. Por ejemplo, cuando alguien me pide el carnet, a pesar de que ya lo tengo por más de un año. Aún así me vienen recuerdos y como que me estremezco. Cuando alguien... por ejemplo, hay una persona con mi nombre anterior y otra persona lo llama por su nombre y dice ese nombre en voz alta, y sobre todo si no lo escuch y y lo dice varias veces, yo empiezo a temblar de puros recuerdos. No quiero escuchar ese nombre, no quiero revivir ese pasado" (TF (26) CON).

Para Pachankis \& Goldfried (2006), la ansiedad es una estrategia frecuente de defensa y/o anticipación frente una amenaza al autoconcepto. De esta forma, constatamos que eventos de la vida cotidiana para cualquier persona cisgénero como presentar la identificación (cédula de identidad) o ser llamado en un lugar público por el nombre, se transforman para las personas transgénero en situaciones que producen altos niveles de ansiedad anticipatoria y angustia, en tanto, se generan expectativas de ser agredidos o violentados, lo cual es definido por el MEM como expectativa de rechazo. "Como habrá sido que los demás me molestaron que hasta el otro loco me quedó mirando y me dijo "oye en todo caso este igual parece mina", molestando... pero yo... preocupado pensando: estos me tiran para adentro, me violan entre todos y no cuento la historia" (TM (28) CON).

En ese sentido, la permanente experimentación de ansiedad genera sentimientos de inseguridad e inadecuación. "Me ha pasado en todos los lugares, o como de estar en playa con alguien y sentir la incomodidad de que hay alguien que está mirando todo el tiempo... como esa inseguridad de saber que me puede pasar algo" (TM (31) VAL). Por lo que resulta significativo remarcar que esta sintomatología se vive en soledad y sin la percepción de apoyo social, como lo vemos en el siguiente relato. "En ese momento no le contaba a nadie, porque como que me daba miedo. O sea, tenía un miedo arraigado de la religión, de lo que opinaba tu familia, de escuchar todos los comentarios de la gente" (TM (25) STGO). 
Con relación al párrafo anterior, la actitud de alerta constante producida por el prejuicio sexual, genera manifestaciones de sintomatología ansiosa, la cual puede escalar y complejizarse mediante cuadros más complejos de salud mental, como es el caso de la depresión, existiendo labilidad y desajuste emocional en el día a día. "De repente como que llegai a tu casa ya deshecha porque es una lucha constante (...) Y te poní a llorar" (TF (35) VAL).

Los trastornos del ánimo son altamente descritos en la literatura referida a los efectos del prejuicio sexual, puesto que se asocian a la aparición de cuadros depresivos de mayor complejidad (Hoffman, 2014). El siguiente relato permite ilustrar dicha vivencia. "Empecé a tener muchas depresiones, crisis de ansiedad, ansiedad sostenida que me duró años y que fue uno de los periodos más difíciles de mi vida” (TF (26) CON).

Las personas transgénero entrevistadas refieren como una experiencia común, la vivencia de sintomatología depresiva, se manifiesta en dos vertientes. Primero, asociada a cambios anímicos ligados a los procesos de hormonización y, segundo, producto del maltrato recibido y percibido. En el caso de la terapia de reemplazo hormonal, esta trae consigo efectos en la salud mental que van desde cambios de humor, irritabibilidad y sintomatología depresiva. Además, somnolencia, disminución del deseo sexual, dolor corporal, así como también, riesgos de producir cáncer. "Los cambios se vienen con síndromes, estos cambios de humor y bajón de ánimo terrible. Yo soy súper cambiante, súper bipolar” (TM (22) AFTA).

De manera que ante la emergencia de sintomatología depresiva, como consecuencia de la violencia recibida, se identifica que existe un estado emocional deteriorado, el cual se retroalimenta en la vida cotidiana por la percepción de discriminación proveniente desde diversos contextos. "Entré en una depresión terrible porque dejar de ver a tu familia, que es el núcleo más cercano que había tenido siempre... porque además, obviamente, no podía tener muchas amistades porque me las negaban. Por lo tanto, mi único círculo más cercano que tenía era mi familia, entonces cuando mi propia madre decide que vamos a tener que inventar una excusa, me dijo: "vamos a decirle a tus tatas que viajaste al extranjero y que no vas a volver más para que ellos no se enteren de que tienen un nieto nuevo", realmente eso fue triste" (TM (27) CON).

Dicho estado es referido desde significaciones ligadas a falta de recursos comunitarios e institucionales a los cuales poder acceder debido a la precariedad económica y laboral existente. Por ejemplo, la dificultad para consultar y temor para asistir a solicitar apoyo a un profesional en un centro de salud pública. Por ende, la gestión de estos procesos a nivel psicológico se desarrolla bajo la falta de recursos que permitan una solución efectiva al problema. Entre las estrategias utilizadas para afrontar la sintomatología depresiva se incluyen la represión y negación de la violencia, a fin de tolerar y/o enfrentar la discriminación percibida. "Me acuerdo un poco. Pero hay cosas que increiblemente se me han ido olvidando, muchas cosas he olvidado, hartas he olvidado, hartas cosas así, caleta de detalles, porque igual pasé caleta... de las de quico y caco(*) que llaman así... como que se me han olvidado, como que siento que empecé y no sé si es algo emocional, no sé, o si la testosterona hizo un efecto. He olvidado mucho... mucho" (TM (29) VAL).

En un ámbito más complejo, los indicadores de suicidalidad en personas LGBT son altos. A nivel internacional, se ha reportado mayores niveles de ideación suicida, intento de suicidio y de suicidios efectivos en dicha población (Haas et al., 2011). También, estudios en personas transgénero han identificado que un tercio de los encuestados informaron haber intentado suicidarse una o más veces (Kenagy, 2005). Por lo que, el siguiente relato ilustra dicha vivencia en la percepción colectiva de la comunidad transgénero. "Tú cuentas que tienes ese problema a cualquier persona y lo único que hacen es discriminarte, decirte que eso está mal, que tú estás mal. Entonces, al final te forman un caldo de cabeza tan brutal que tú colapsas, tú caes. En ese momento no sabes que hacer y no me extraña que la gente piense en el suicidio. Yo lo pensé mucho tiempo. No me extraña que la gente se suicide porque de verdad es un infierno. De verdad es muy doloroso, es una cosa horrible" (TF (26) CON).

De esta forma, desde la comunidad transgénero, el suicidio es identificado como un fenómeno altamente prevalente en dicho grupo y una forma de escape frente a la desesperanza. "Y te llevan nuevamente a caer en depresiones y en problemas psicológicos que son bastante complicados, después de tener años de acumular ciertas cosas. Te cuesta un poco levantarte de ahí, porque se va acumulando una cantidad de cosas negativas en tu vida y llega un punto en el que cuesta mucho salir de ahi. Y si no se tiene la ayuda necesaria... conozco muchos chicos que han terminado suicidándose, porque no han tenido la ayuda que han necesitado en el momento preciso" (TM (27) STGO).

Las personas entrevistadas significan que el suicidio puede ser — en algunos casos — una estrategia para gestionar los altos niveles de prejuicio sexual experimentado en lo cotidiano y la ansiedad consecuente. "Mi 
madre me negó ver a mis abuelos, fue tremendo, fue muy muy malo, muy negativo, y lamentablemente, me acarreó una depresión bastante grande, intentos de suicidio, obviamente entre medio, que es muy común en la población trans (...) uno obviamente no tiene las herramientas cerca para poder salir del problema" (TM (27) $\mathrm{CON}$ ). Tales hallazgos van en sintonía con lo reportado por distintas investigaciones sobre suicidio en minorías sexuales y de género (Tomicic et al., 2016), y específicamente, en población transgénero (Clements-Nolle, Marx \& Kartz, 2006; Grossman \& D'Augelli, 2008).

En última instancia, la aparición de conductas autolesivas y consumo de sustancias, como manifestación de trastornos ligados a la salud mental de población transgénero, ha sido reportado por la literatura internacional (Jackman, Honig, \& Bockting, 2016). Este comportamiento podría estar relacionado con el estrés vivido durante el proceso de afirmación de género, a la vez que en el enfrentamiento de la hostilidad y rechazo (Jackman, Honig, \& Bockting, 2016). Respecto a este punto, las personas entrevistadas relatan la experimentación de conductas autolesivas relacionadas al desajuste emocional por el que atraviesan en su cotidianeidad las personas transgénero. "Estaba ahí la Gillette con la que a veces me afeitaba (...) la rompí y me empecé a cortar. Lo único que necesitaba era salir de la situación y creo que busqué lo que en algún momento me sirvió. No se como uno llega a ese extremo, porque la mente se te quiebra. Realmente sentí eso. Recuerdo que tenía todo el brazo sangrando" (TM (28) CON).

En otro sentido, una estrategia de afrontamiento reportada por las personas transgénero participantes fue que con frecuencia existe consumo de sustancias, entre las que destacan el alcohol y otras drogas recreativas. El consumo de alcohol es referido como una forma de evitación y evasión del entorno, "y copete, copete, copete. Full copete, tenía un consumo muy problemático" (TM (28) CON). Asimismo, se identifica el uso de diversas drogas que ponen en riesgo tanto la salud mental como física de las personas transgénero, "empezamos a meternos a las drogas, así como full porro prensado, raspado de murallas, neopren, copete (...) ya no era para conocer Santiago, si no que era para drogarse, tomar ... chao, evadir, evadir, evadir” (TM (28) CON).

\section{Discusión}

Las experiencias analizadas refieren la experimentación de un mayor nivel de estrés social en la población transgénero, lo cual afectaría directamente su salud mental. Dicho estrés es provocado por el estigma y el prejuicio sexual presente en la población general y orientado hacia las personas transgénero.

En este sentido, en los relatos se identifican el impacto tanto de los estresores distales (por ejemplo, eventos de discriminación), así como proximales (por ejemplo, transfobia internalizada) asociados de forma persistente a la trayectoria vital de las personas transgénero.

Por otro lado, la constante violencia debido al prejuicio sexual hacia las personas transgénero posibilitaría la aparición y desarrollo de una evaluación negativa del sí mismo, experimentando como consecuencia diversas reacciones emocionales. En ese sentido, la corporalidad de las personas transgénero emerge de forma física y simbólica como un espacio donde se experimentan el estigma y el prejuicio sexual, al afectar de forma sistemática la construcción de su propio autoconcepto (Salazar, 2014).

Las personas entrevistadas describen como la expectativa de rechazo se manifiesta como una reacción de anticipación de un eventual hecho de discriminación debido a elementos identitarios. Esto es clave para comprender el impacto que tiene el prejuicio sexual en la población transgénero, y con ello, sostener la utilidad metodológica del MEM para dar cuenta de la vivencia diferenciada del estrés en la población transgénero en comparación con la población general (Hendricks \& Testa, 2012; Meyer, 2003).

Con relación a los antecedentes presentados, podemos concluir que la salud mental de las personas transgénero es baja y deteriorada debido a los efectos del prejuicio sexual. La presencia de sintomatología ansiosadepresiva, la ideación e intento suicida y el consumo problemático de alcohol y otras sustancias reportadas en este estudio son una evidencia de la manera en que impacta el contexto social y cultural en la vida de las personas transgénero.

Con base en lo anterior, emerge la necesidad de preguntarse cómo acompañar desde la disciplina psicológica a las personas transgénero en su cotidianeidad, desde una mirada alejada de psicopatologización de su identidad, y precisamente, desde el reconocimiento del impacto del prejuicio sexual en su salud mental (RosellóPeñaloza, 2018). 
De este modo, se facilita una comprensión de forma integral de las dificultades percibidas por las personas transgénero y se contribuye a disminuir los efectos negativos y el deterioro de su salud mental. Asimismo, se pueden implementar estrategias sensibles a los recursos personales de la población transgénero a partir del reconocimiento de diversos estresores sociales (Martínez et al., 2018; Tomicic et al., 2016).

A modo de discusión final, resulta fundamental transmitir a la comunidad académica y de profesionales que trabajan en la atención de personas transgénero que una salud mental positiva de este colectivo se encuentra asociada a: la aceptación y reconocimiento de su identidad, abriendo un debate a la no patologización; el buen trato en diversos contextos sociales, favoreciendo la integración de personas transgénero en sus entornos; y, finalmente, el apoyo de pares y familiares, lo cual les reporta bienestar, seguridad y aceptación.

\section{Limitaciones}

La unidad de análisis de este estudio abarca participantes, en su mayoría, personas transgéneros binarias y mayores de 21 años. En estudios futuros, sería conveniente incorporar a personas con identidades no binarias y de otros rangos de edad, a fin de ampliar y enriquecer la comprensión de la identidad transgénero. Asimismo, indagar sobre las implicaciones y el impacto de la transición social y médica en la trayectoria vital de las personas transgénero.

En otro sentido, estudios futuros deberían incorporar análisis que contemplen cómo el prejuicio, en algunas circunstancias, no solo produce efectos negativos sino que también, produce crecimiento. En este sentido, se requieren estudios futuros que aborden fenómenos de resiliencia en esta poblaciones. En ese aspecto, los resultados de esta investigación nos muestran la generación de estrategias de afrontamiento individuales y colectivas a la violencia, las cuales no podrán ser desarrolladas debido a la extensión de esta comunicación y serán reportadas en un artículo posterior.

\section{Implicaciones}

Reisner y colaboradores (2016), sugieren poner a prueba los modelos teóricos existentes que intentan explicar las disparidades en salud entre diversos tipos de poblaciones, o bien, desarrollar nuevos modelos que permitan guiar la investigación relativa a indicadores positivos de salud y bienestar en la población transgénero.

Tal como lo indicamos, se han aplicado varios modelos conceptuales, entre ellos el MEM. Este modelo y otros reconocen la existencia de múltiples niveles entrecruzados en la definición del riesgo y resiliencia que moldean la salud y bienestar de las personas transgénero, concordando en que es siempre necesario un abordaje multinivel contextualmente relevante (Reisner et al., 2016).

Por otro lado, la entrada en vigencia de la ley de identidad de género en el país, supone una serie de desafíos para instituciones educativas, de salud, y asimismo, la sociedad en su conjuto. Por tanto, en el corto y mediano plazo la implementación de dicha ley supone la realización de cambios respecto de procedimientos institucionales, además de la educación a la sociedad civil sobre las identidades transgénero. En particular, si bien ya existen reglamentos respecto a la atención de personas transgénero en el sistema de salud y escolar, en dichos espacios precisamente habrá que poner particular atención para disminuir las barreras de acceso a dichos sistemas.

Desde un punto de vista disciplinar, aún persiste la patologización de las personas transgénero, lo que es ejecutado por muchos médicos endocrinólogos, psiquiatras y psicólogos. La aplicación del diagnóstico de "disforia de género" supone una enorme barrera para la disminución del estigma y el prejuicio hacia las personas transgénero. En consonancia con lo anterior, la formación universitaria en carreras concernidas debe estimular la despatologización y el acercamiento a las identidades transgénero desde una óptica de respeto y resguardo de sus derechos. Sin duda, lo anterior no es sencillo. La permanencia en el principal manual diagnóstico de enfermedades mentales (DSM) de la categoría "disforia de género" constituye una barrera y una forma de violencia estructural de difícil manejo. Por ende, la implementación de psicoterapias sensibles culturalmente a las minorías sexuales y de género es fundamental. Dichas psicoterapias deben facilitar los procesos de afirmación de género sin contribuir a re-patologizar las identidades.

Finalmente, no olvidemos que todos los desafíos e implicaciones anteriores pueden verse amenazadas debido al avance sostenido de los movimientos anti-género en el mundo y en el país (Corrêa, 2018). Dichos movimientos, promueven denominar a la agenda ligada a temáticas y reivindicaciones feministas y de la diversidad sexual 
como "ideología de género", luchando tanto en el parlamento de algunos países, como en diversos espacios de la sociedad civil, contra la noción misma de género y uno de sus principales focos de ataque y denostación son precisamente las personas transgénero y la consecuión de sus derechos que por años han sido vulnerados y relegados a nivel gubernamental y social .

\section{Referencias}

American Psychological Association. [APA] (2015). Guidelines for psychological practice with transgender and gender nonconforming people. American Psychologist, 70, 832-864. doi: 10.1037/a0039906

Barrientos, J. (2015). Violencia homofóbica en América Latina y Chile. Santiago, Editorial el Buen Aire.

Barrientos, J., Cárdenas, M., Gómez, F., \& Guzmán, M. (2016). Gay men and male-to-female transgender persons in Chile: an exploratory quantitative study on stigma, discrimination, victimization, happiness and social well-being: pp. 253-270. En: Thomas Köllen (ed). Sexual Orientation and Transgender Issues in Organizations - Global Perspectives on LGBT Workforce Diversity. Sage Publications. doi: https://doi.org/10.1007/978-3-319-29623-4_15

Berredo, L. (2011). Dificultades administrativas enfrentadas por las personas trans en la región Metropolitana de Chile (tesis pregrado). Santiago: Universidad Academia de Humanismo Cristiano.

Brito, A. (Coord.) (2019). Violencia extrema, los asesinatos de personas LGTBI en México: Los saldos del sexenio (2013-2018). México: Letra $\mathrm{S}$.

Bockting, W., Coleman, E., Deutsch, M. B, Guillamon, A., Meyer, I., Meyer, W., Reisner, S., Sevelius, J., \& Ettner, R. (2016). Adult development and quality of life of transgender and gender nonconforming people. Current Opinion in Endocrinology, Diabetes and Obesity, 23, 188-197. doi: 10.1097/MED.0000000000000232

Calton, J., Bennett, L., \& Gebhard, K. (2016). Barriers to Help Seeking for Lesbian, Gay, Bisexual, Transgender, and Queer Survivors of Intimate Partner Violence. Trauma, Violence \& Abuse, 17, 585-600. doi: https://doi.org/10.1177/1524838015585318

Casanova, P., \& Espinoza-Tapia, R. (2018). Significados en torno a la atención psicológica durante el proceso de transición de género: una aproximación desde la perspectiva de usuarios/as transgénero en Chile. Al Sur de todo, 12, 201-228.

Comisión Interamericana de Derecho Humanos [CIDH] (2015). Violencia contra personas lesbianas, gais, bisexuales, trans e intersex en América. OAS/Ser.L/V/ II.rev.2. Recuperado desde http://bit.ly/2vyAKoV.

Clements-Nolle, K., Marx, R., Guzman, R., \& Katz, M. (2001). HIV prevalence, risk behaviors, health care use, and mental health status of transgender persons: implications for public health intervention. American journal of public health, 91, 915-921. doi:10.2105/ajph.91.6.915

Clements-Nolle K., Marx R., \& Katz M. (2006). Attempted suicide among transgender persons: The influence of gender-based discrimination and victimization. Journal of Homosexuality, 51, 53-69. doi: 10.1300/J082v51n03_04

Corrêa, S. (2018). Ideología de Género: rastreando sus orígenes y significados en la política de género actual. Sexuality, Policy, Watch [online]. Recuperado desde https://sxpolitics.org/es/ideologia-de-genero-rastreando-sus-origenes-y-significados-en-la-politica-degenero-actual $/ 3858$

Coll-Planas, G. (2010). La voluntad y el deseo. La construcción social del género y la sexualidad: El caso de lesbianas, gays y trans. Barcelona/Madrid: Egales.

Charmaz, K. C. (2006). Constructing Grounded Theory: A Practical Guide Through Qualitative Analysis. Thousand Oaks, CA: pp. $284-320$.

Davidson, M. (2007). Seeking refuge under the umbrella: Inclusion, exclusion, and organizing within the category transgender. Sexuality Research and Social Policy, 4, 60-80. doi:10.1525/srsp.2007.4.4.60

Dhejne, C., Van Vlerken, R., Heylens, G., \& Arcelus, J. (2016) Mental health and gender dysphoria: A review of the literature. International Review of Psychiatry, 28, 44-57. doi:10.3109/09540261.2015.1115753

Fraïssé, C., \& Barrientos, J. (2016). La notion d'homophobie: une approximation psychosociale. Sexologies, 25, 133-140. doi: 10.1016/j.sexol Glasser, B., \& Strauss, A. (1967). The Discovering Grounded Theroy: strategies for qualitive research. New York: Aldine.

Grossman, A.H., \& D’Augelli, A. R. (2008). Transgender youth and life threatening behaviors. Suicide and Life Threatening Behavior, 37, 527-537.

Haas, A.P., Eliason, M., Mays, V.M., Mathy, R. M., Cochran, S. D., D’Augelli, AR,... Clayton, P. J. (2011) Suicide and suicide risk in lesbian, gay, bisexual, and transgender populations: review and recommendations. Journal of Homosexuality, 58, 10-51. doi: 10.1080 / 00918369.2011.534038

Hendricks, M. L., \& Testa, R. J. (2012). A conceptual framework for clinical work with transgender and gender nonconforming clients: An adaptation of the Minority Stress Model. Professional Psychology: Research and Practice, 43, 460-467. doi: http://dx.doi.org/10.1037/ a0029597 
Herek, G. M., Gillis, J. R., \& Cogan, J. C. (2009) Internalized stigma among sexual minority adults: Insights from a social psychological perspective. Journal of Counseling Psychology, 56, 32-43. doi:10.1037/a0014672

Herek, G. M. (2000) The Psychology of Sexual Prejudice. Current Directions in Psychological Science, 9, 19-22. doi: https://doi. org/10.1111/1467-8721.00051

Hoffman, B. (2014). An Overview of Depression among Transgender Women. Depression Research and Treatment, vol 2014. doi: https:// doi.org/10.1155/2014/394283.

Jackman, K., Honig, J., \& Bockting, W. (2016). Nonsuicidal self-injury among lesbian, gay, bisexual and transgender populations: an integrative review. Journal of Clinical Nursing, 25, 3438-3453. doi:10.1111/jocn.13236

Kenagy, G. P. (2005). Transgender health: Findings from two needs assessment studies in Philadelphia. Health \& Social Work, 30, 19-26. doi: $10.1093 / \mathrm{hsw} / 30.1 .19$

Mathy, R. M. (2002). A nonclinical comparison of transgender identity and sexual orientation: a framework for multicultural competence. Journal of Psychology \& Human Sexuality, 13, 31-54. doi: 10.1300/J056v13n01_02

Martínez, C., Tomicic, A., Gálvez, C., Rodríguez, J. Rosenbaum, C., \& Aguayo, F. (2018). Psicoterapia Culturalmente Competente para el Trabajo con Pacientes LGBT+. Una Guía para Psicoterapeutas y Profesionales de la Salud Mental. Santiago, Chile: Centro de Estudios en Psicología Clínica \& Psicoterapia, Universidad Diego Portales (CEPPS-UDP).

Meyer, I. H. (2003). Prejudice, Social Stress, and Mental Health in Lesbian, Gay, and Bisexual Populations: Conceptual Issues and Research Evidence. Psychological Bulletin, 129, 674-697. doi: http://doi.org/10.1037/0033-2909.129.5.674

MINISTERIO DE SALUD DE CHILE. (2017). Plan nacional de salud mental 2017-2025. Recuperado desde https://www.minsal.cl/ wp-content/uploads/2017/12/PDF-PLAN-NACIONAL-SA LUD-MENTAL-2017-A-2025.-7-dic-2017.pdf

Movimiento Unificado de Minorías Sexuales [MUMS] (2009). Caracterización de la vulnerabilidad individual y grupal de personas trans de la Región Metropolitana, con énfasis en aquellas que ejercen el comercio sexual. Santiago de Chile. Recuperado desde http://www. scribd.com/doc/113015091/

Organizando Trans Diversidades (2017). Informe Ejecutivo Encuesta-T. Santiago: OTD. Recuperado desde encuesta-t.cl

Oficina de las Nacionales Unidad contra la Droga y el Delito [UNODC]. (2019). Nada que curar. Guia de referencia para profesionales de la salud mental en el combate a los ECOSIG (Esfuerzos para Corregir la Orientación Sexual y la Identidad de Género). Recuperado desde https://www.unodc.org/documents/mexicoandcentralamerica /2019/GUIAECOSIGFINAL-compressed.pdf

Organización Panamericana de la Salud [OPS]. (2016). Por la salud de las personas trans. Elementos para el desarrollo de la atención integral de personas trans y sus comunidades en Latinoamérica y el Caribe. Recuperado desde http://www.paho.org/arg/images/gallery/ Blueprint $\% 20$ Trans $\% 20$ Espa $\tilde{ \pm} \pm$ ol.pdf

Patton, M. (2002). Qualitative Research \& Evaluation Methods. Thousand Oaks, Estados Unidos: Sage Publications.

Pérez-Serrano, G. (1998). Investigación Cualitativa: Retos e Interrogantes Vol.1. Madrid: La Muralla.

Pachankis, J. E., \& Goldfried, M. R., (2006). Social anxiety in young gay men, Journal of Anxiety Disorders, 20, 996-1015. doi: 10.1016/j. janxdis.2006.01.001

Pachankis, J.E. (2016). Discriminatory experiences associated with posttraumatic stress disorder symptoms among transgender adults. Journal of counseling psychology, 63, 5, 509-519. 509. doi: 10.1037/cou0000143

Reisner, S. L., White Hughto, J. M., Gamarel, K. E., Keuroghlian, A. S., Mizock, L., \& Pachankis, J.E. (2016). Discriminatory experiences associated with posttraumatic stress disorder symptoms among transgender adults. Journal of Counseling Psychology, 63(5), 509-519. doi: https://doi.org/10.1037/cou0000143

Roselló-Peñaloza, M. (2018). NO BODY. Clinical Constructions of Gender and Transsexuality-Pathologization, Violence and Deconstruction. London: Routledge.

Salazar, X (2014). Vine al mundo porque Dios quiere que yo esté aquí: recorridos identitarios de mujeres trans en Lima, Iquitos y Ayacucho (Tesis doctoral). Pontificia Universidad Católica del Perú. Recuperado desde http://hdl.handle.net/20.500.12404/6720

Tomicic, A., Gálvez, C., Quiroz, C., Martínez, C., Fontbona, J., Rodríguez, J., Aguayo, F., Rosenbaum, C., Leyton, F., \& Lagazzi, I. (2016). Suicidio en poblaciones lesbiana, gay, bisexual y trans: revisión sistemática de una década de investigación (2004-2014). Revista médica de Chile, 144, 723-733. doi: https://dx.doi.org/10.4067/S0034-98872016000600006

Valentine, D. (2007) Imagining Transgender: An Ethnography of a Category. Durham, NC: Duke University Press: pp. 27-68. 
